\title{
PLURICANONICAL MAPS OF VARIETIES OF MAXIMAL ALBANESE DIMENSION
}

\author{
Jungkai A. Chen, Christopher D. Hacon
}

April 27, 2000

\begin{abstract}
Let $X$ be a smooth complex projective algebraic variety of maximal Albanese dimension. We give a characterization of $\kappa(X)$ in terms of the set $V^{0}\left(X, \omega_{X}\right)$ $:=\left\{P \in \operatorname{Pic}^{0}(X) \mid h^{0}\left(X, \omega_{X} \otimes P\right) \neq 0\right\}$. An immediate consequence of this is that the Kodaira dimension $\kappa(X)$ is invariant under smooth deformations. We then study the pluricanonical maps $\varphi_{m}: X \rightarrow \mathbb{P}\left(H^{0}\left(X, m K_{X}\right)\right)$. We prove that if $X$ is of general type, $\varphi_{m}$ is generically finite for $m \geq 5$ and birational for $m \geq 5 \operatorname{dim}(X)+1$. More generally, we show that for $m \geq 6$ the image of $\varphi_{m}$ is of dimension equal to $\kappa(X)$ and for $m \geq 6 \kappa(X)+2, \varphi_{m}$ is the stable canonical map.
\end{abstract}

\section{INTRODUCTION}

Generic vanishing theorems have recently proven to be a very effective tool in the study of the geometry of irregular varieties. In this paper we will show how these techniques can be used to answer a series of natural questions about varieties of maximal Albanese dimension.

In what follows, $X$ will denote a smooth complex projective algebraic variety, $\operatorname{Alb}(X)$ denotes the Albanese variety of $X$ and $\operatorname{alb}_{X}: X \longrightarrow \operatorname{Alb}(X)$ the corresponding Albanese map. We will assume that $X$ is of maximal Albanese dimension and hence $\mathrm{alb}_{X}$ is a generically finite morphism. In particular $q(X) \geq \operatorname{dim}(X)$. Kollár made the following

Conjecture $[\mathrm{Ko} 3,(17.9 .3)]$. If $X$ is of general type and maximal Albanese dimension. Then $\chi\left(X, \omega_{X}\right)>0$.

As an immediate consequence of the generic vanishing theorems of Green and Lazarsfeld (cf. Theorem 1.3.1), one sees that this is equivalent to $h^{0}\left(X, \omega_{X} \otimes P\right)>$ 0 for all $P \in \operatorname{Pic}^{0}(X)$. In [EL1, Example 1.13], Ein and Lazarsfeld produce a counterexample to this conjecture. However, we will show that a similar result does hold.

The first author was partially supported by National Science Council, Taiwan (NSC-89-2115M-194-016)

1991 Mathematics Subject Classification. Primary 14C20, 14F17. 
Theorem 1. The translates through the origin of the irreducible components of $V^{0}\left(X, \omega_{X}\right):=\left\{P \in \operatorname{Pic}^{0}(X) \mid h^{0}\left(X, \omega_{X} \otimes P\right) \neq 0\right\}$ generate a subvariety of $\operatorname{Pic}^{0}(X)$ of dimension $\kappa(X)-\operatorname{dim}(X)+q(X)$. In particular, if $X$ is of general type, $V^{0}\left(X, \omega_{X}\right)$ generates $\operatorname{Pic}^{0}(X)$.

This is a generalization of a result of Ein and Lazarsfeld [EL2] (see Theorem 1.3.2). An immediate consequence is the following

Corollary 2. Let $X$ be of maximal Albanese dimension. Then the Kodaira dimension of $X$ is invariant under smooth deformations.

We next turn our attention to the study of the linear series $\left|m K_{X}\right|$ and the corresponding rational maps $\varphi_{m}:=\varphi_{\left|m K_{X}\right|}$. In [Ko3], Kollár shows that if $X$ is smooth proper variety of general type with generically large algebraic fundamental group, then the sections of $\left|m K_{X}\right|$ define a birational map for $m \geq 10^{\operatorname{dim}(X)}$. Following $[\mathrm{AS}]$ it is reasonable to expect a quadratic bound in $\operatorname{dim}(X)$.

Theorem 3. If $X$ is of maximal Albanese and Kodaira dimensions. Then $\varphi_{5}$ is generically finite, and $\varphi_{5 \operatorname{dim}(X)+1}$ is birational.

Our proof is based on the techniques of Kollár [Ko3]. However, technical difficulties imply that when $X$ is not of general type, the bounds are somewhat weaker.

Theorem 4. If $X$ is of maximal Albanese dimension. Then $\varphi_{6}(X)$ has dimension equal to $\kappa(X)$ and $\varphi_{6 \kappa(X)+2}$ is the stable canonical map.

We do not expect these bounds to be optimal. It would be interesting to produce examples of varieties of general type and maximal Albanese dimension for which $\varphi_{m}$ is not birational for $m \geq 3$.

Acknowledgment. We would like to thank P. Belkale, H. Clemens, Y. Kawamata, J. Kollár, R. Lazarsfeld and I.-H. Tsai for valuable conversations.

\section{Conventions and Notations}

(0.1) Throughout this paper, we work over the field of complex numbers $\mathbb{C}$.

(0.2) For $D_{1}, D_{2} \mathbb{Q}$-divisors on a variety $X$, we write $D_{1} \prec D_{2}$ if $D_{2}-D_{1}$ is effective, and $D_{1} \equiv D_{2}$ if $D_{1}$ and $D_{2}$ are numerically equivalent.

(0.3) $|D|$ will denote the linear series associated to the divisor $D$, and $B s|D|$ denotes the base locus of $|D|$.

(0.4) For a real number $a$, let $\lfloor a\rfloor$ be the largest integer $\leq a$ and $\lceil a\rceil$ be the smallest integer $\geq a$. For a $\mathbb{Q}$-divisor $D=\sum a_{i} D_{i}$, let $\lfloor D\rfloor=\sum\left\lfloor a_{i}\right\rfloor D_{i}$ and $\lceil D\rceil=\sum\left\lceil a_{i}\right\rceil D_{i}$.

(0.5) Let $\mathcal{F}$ be a coherent sheaf on $X$, then $h^{i}(X, \mathcal{F})$ denotes the complex dimension of $H^{i}(X, \mathcal{F})$. In particular, the plurigenera $h^{0}\left(X, \omega_{X}{ }^{\otimes m}\right)$ are denoted by $P_{m}(X)$ and the irregularity $h^{0}\left(X, \Omega_{X}^{1}\right)$ is denoted by $q(X)$.

(0.6) We will denote by $\operatorname{Alb}(X)$ the albanese variety of $X$, by $\operatorname{alb}_{X}: X \longrightarrow$ $\operatorname{Alb}(X)$ the albanese morphism. As usual $\operatorname{Pic}^{0}(X)$ is the abelian variety dual to $\operatorname{Alb}(X)$ parametrazing all topologically trivial line bundles on $X \cdot \operatorname{Pic}^{\tau}(X)$ will denote the set of torsion elements in $\operatorname{Pic}^{0}(X)$.

(0.7) Let $f: X \rightarrow Y$ be a generically finite morphism. $R_{f}$ denotes the ramification divisor. 
(0.8) Let $f: X \rightarrow Y$ be a morphism. An irreducible divisor $E$ on $X$ is said to be $f$-exceptional if $\operatorname{dim} f(E)<\operatorname{dim} f(X)-1$. And $E$ is $f$-vertical if $\operatorname{dim} f(E) \leq \operatorname{dim} f(X)-1$. An effective divisor is $f$-exceptional (resp. $f$-vertical) if every component is.

(0.9) We will say that a $\mathbb{Q}$-divisor $\Delta$ is klt (Kawamata log terminal) if $\Delta$ has normal crossings support and $\lfloor\Delta\rfloor=0$. We refer to [Ko3, 10.1.5] for the general definition of klt divisor.

\section{Preliminaries}

\subsection{Higher Direct Images of Dualizing Sheaves.}

For the convenience of the reader, we recall various results which will be frequently used in what follows.

Theorem 1.1.1. Let $X, Y$ be projective varieties, $X$ smooth and $f: X \rightarrow Y$ a surjective map. Let $M$ be a line bundle on $X$ such that $M \equiv f^{*} L+\Delta$, where $L$ is $a \mathbb{Q}$-divisor on $Y$ and $(X, \Delta)$ is klt. Then

(a) $R^{j} f_{*}\left(\omega_{X} \otimes M\right)$ is torsion free for $j \geq 0$.

(b) if $L$ is nef and big, then $H^{i}\left(Y, R^{j} f_{*}\left(\omega_{X} \otimes M\right)\right)=0$ for $i>0, j \geq 0$.

(c) $R^{i} f_{*} \omega_{X}$ is zero for $i>\operatorname{dim}(X)-\operatorname{dim}(Y)$.

(d) $R \cdot f_{*} \omega_{X} \cong \sum R^{i} f_{*} \omega_{X}[-i]$ and $h^{p}\left(X, \omega_{X}\right)=\sum h^{i}\left(Y, R^{p-i} f_{*} \omega_{X}\right)$.

(e) If $Y$ is birational to an abelian variety and $L$ is nef and big, it follows that $H^{0}\left(Y, R^{j} f_{*}\left(\omega_{X} \otimes M\right) \otimes P\right)=0$ for all $P \in \operatorname{Pic}^{0}(Y)$ if and only if $R^{j} f_{*}\left(\omega_{X} \otimes M\right)=0$.

Proof. See [Ko1], [Ko2], [Ko3], [Ka2], [EV1].

Theorem 1.1.2. Let $f: X \longrightarrow Y$ be a surjective morphism from a smooth projective variety to a projective variety. Let $Q \in \operatorname{Pic}^{\tau}(X)$. Then

$$
R f_{*}\left(\omega_{X} \otimes Q\right) \cong \sum R^{i} f_{*}\left(\omega_{X} \otimes Q\right)[-i] .
$$

In particular $h^{p}\left(X, \omega_{X} \otimes Q\right)=\sum h^{i}\left(Y, R^{p-i} f_{*}\left(\omega_{X} \otimes Q\right)\right)$.

Proof. [Ko2, Corollary 3.3].

\subsection{The Iitaka Fibration.}

Let $X$ be a smooth complex projective variety of maximal Albanese dimension. Let $a: X \longrightarrow A$ be a generically finite map such that the image of $X$ generates the abelian variety $A$. A nonsingular representative of the Iitaka fibration of $X$ is a morphism of smooth complex projective varieties $f^{\prime}: X^{\prime} \longrightarrow Y$ such that $\operatorname{dim}(Y)=\kappa(X)$ and $\kappa\left(X^{\prime}{ }_{y}\right)=0$, where $X^{\prime}{ }_{y}$ is a generic geometric fiber of $f^{\prime}$. Since our questions will be birational in nature, we may always assume that $X=X^{\prime}$ and $f=f^{\prime}$. Since $\kappa\left(X_{y}\right)=0$, it follows by [Ka1] that the image of the fiber $X_{y}$ is the translate of an abelian subvariety $K_{y}$ of $A$, and $\left.a\right|_{X_{y}}: X_{y} \longrightarrow K_{y}$ is birationally equivalent to an étale map. Since $A$ can contain at most countably many abelian subvarieties, we may assume that the $K_{y}$ are all translates of a fixed abelian subvariety $K$ of $A$. We will denote by $\tilde{K}$ the abelian variety birational to $X_{y}$. Let $p: A \longrightarrow S:=A / K$. Let $Z$ (resp. $W$ ) denote the image of $X$ in $A$ (resp. $S$ ). By construction, the general fiber of $f: X \longrightarrow Y$ maps to a closed point in $S$, therefore replacing $X$ by an appropriate birational model, there exists a morphism 
$q: Y \longrightarrow S$ such that $q \circ f=p \circ a$. We may assume that the above maps fit in the following commutative diagram

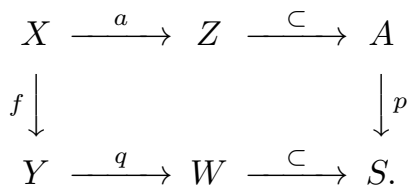

\subsection{Cohomological Support Loci.}

Let $a: X \rightarrow A$ be a morphism from a smooth projective variety $X$ to an abelian variety $A$. If $\mathcal{F}$ is a coherent sheaf on $X$, then one can define the cohomological support loci by

$$
V^{i}(X, T, \mathcal{F}):=\left\{P \in T \subset \operatorname{Pic}^{0}(A) \mid h^{i}\left(X, \mathcal{F} \otimes a^{*} P\right) \neq 0\right\} .
$$

In particular, if $a=\operatorname{alb}_{X}: X \rightarrow \operatorname{Alb}(X)$, then we simply write

$$
V^{i}(X, \mathcal{F}):=\left\{P \in \operatorname{Pic}^{0}(X) \mid h^{i}(X, \mathcal{F} \otimes P) \neq 0\right\} .
$$

We say that $X$ has maximal Albanese dimension if $\operatorname{dim}\left(\operatorname{alb}_{X}(X)\right)=\operatorname{dim}(X)$. The geometry of the loci $V^{i}\left(X, \omega_{X}\right)$ defined above is governed by the following:

\section{Theorem 1.3.1 (Generic vanishing).}

(a) Any irreducible component of $V^{i}\left(X, \omega_{X}\right)$ is a translate of a sub-torus of $\operatorname{Pic}^{0}(X)$ and is of codimension at least $i-\left(\operatorname{dim}(X)-\operatorname{dim}\left(\operatorname{alb}_{X}(X)\right)\right)$.

(b) Let $P$ be a general point of an irreducible component $T$ of $V^{i}\left(X, \omega_{X}\right)$. Suppose that $v \in H^{1}\left(X, \mathcal{O}_{X}\right) \cong T_{P} \operatorname{Pic}^{0}(X)$ is not tangent to $T$. Then the sequence

$$
H^{i-1}\left(X, \omega_{X} \otimes P\right) \stackrel{\cup v}{\longrightarrow} H^{i}\left(X, \omega_{X} \otimes P\right) \stackrel{\cup v}{\longrightarrow} H^{i+1}\left(X, \omega_{X} \otimes P\right)
$$

is exact. If $v$ is tangent to $T$, then the maps in the above sequence vanish.

(c) If $X$ is a variety of maximal Albanese dimension, then

$$
\operatorname{Pic}^{0}(X) \supset V^{0}\left(X, \omega_{X}\right) \supset V^{1}\left(X, \omega_{X}\right) \supset \ldots \supset V^{n}\left(X, \omega_{X}\right)=\left\{\mathcal{O}_{X}\right\} .
$$

(d) Every irreducible component of $V^{i}\left(X, \omega_{X}\right)$ is a translate of a sub-torus of $\operatorname{Pic}^{0}(X)$ by a torsion point.

Proof. See [GL1],[GL2],[EL1] and [S].

In [EL1], Ein and Lazarsfeld illustrate various examples in which the geometry of $X$ can be recovered from information on the loci $V^{i}\left(X, \omega_{X}\right)$. In particular, they prove

Theorem 1.3.2 [EL2]. If $X$ is a variety with maximal Albanese dimension and $\operatorname{dim} V^{0}\left(X, \omega_{X}\right)=0$. Then $X$ is birational to an abelian variety.

Proposition 1.3.3 [EL2]. Let $a: X \longrightarrow A$ be a generically finite map from a smooth projective variety to an abelian variety. Let $P$ be any isolated point of $V^{0}\left(X, \operatorname{Pic}^{0}(A), \omega_{X}\right)$. Then $a^{*} P=\mathcal{O}_{X}$.

We will also need the following

Lemma 1.3.4 [CH, Lemma 2.1]. Let $X$ be a variety of maximal Albanese dimension. Fix $Q \in \operatorname{Pic}^{\tau}(X)$. Then $h^{0}\left(X, \omega_{X}^{2} \otimes Q \otimes f^{*} P\right)$ is constant for all torsion $P \in \operatorname{Pic}^{0}(Y)$. 


\section{KodAira dimension of VARIEties of maximal Albanese Dimension}

Throughout this paper, we will assume that $X$ is of maximal Albanese dimension and hence of positive Kodaira dimension $\kappa(X) \geq 0$. We will need the following immediate consequence of [M, Theorem 2.2]

Lemma 2.1. If $\mathcal{F}$ is a coherent sheaf on an abelian variety $A$ such that $h^{i}(A, \mathcal{F} \otimes P)$ $=0$ for all $i \geq 0$ and all $P \in \operatorname{Pic}^{0}(A)$. Then $\mathcal{F}=0$.

We will frequently refer to the notation and results of $\S 1$. Define

$$
G:=\operatorname{ker}\left(\operatorname{Pic}^{0}(A) \longrightarrow \operatorname{Pic}^{0}(K) \longrightarrow \operatorname{Pic}^{0}(\tilde{K})\right) .
$$

Let $\bar{G}:=G / \operatorname{Pic}^{0}(S)$. By dimension considerations $\bar{G}$ is a finite group, hence $G$ consists of finitely many translates of $\operatorname{Pic}^{0}(S)$. Let $Q_{1}, \ldots, Q_{r} \in G \subset \operatorname{Pic}^{0}(A)$ be a set of torsion line bundles representing lifts of the elements of $\bar{G}$.

We will frequently identify $\operatorname{Pic}^{0}(S)$ with its image in $\operatorname{Pic}^{0}(A)$.

\section{Lemma 2.2.}

(a) $V^{0}\left(X, \operatorname{Pic}^{0}(A), \omega_{X}\right) \subset G$.

(b) For every $Q_{i}$ the loci $V^{0}\left(X, Q_{i}+\operatorname{Pic}^{0}(S), \omega_{X}\right)$ are nonempty.

(c) If $\mathcal{O}_{X} \notin a^{*}\left(Q_{i}+\operatorname{Pic}^{0}(S)\right)$, then there exists a positive dimensional component of $V^{0}\left(X, Q_{i}+\operatorname{Pic}^{0}(S), \omega_{X}\right)$.

Proof. If $H^{0}\left(X, \omega_{X} \otimes a^{*} P\right) \neq 0$ for some $P \in \operatorname{Pic}^{0}(A)$, then for general $y \in Y$, $h^{0}\left(X_{y}, \omega_{X_{y}} \otimes a^{*} P\right)=h^{0}\left(\tilde{K}, \mathcal{O}_{\tilde{K}} \otimes a^{*} P\right) \neq 0$. This is possible only if $P$ is in the kernel of $\operatorname{Pic}^{0}(A) \longrightarrow \operatorname{Pic}^{0}(\tilde{K})=\operatorname{Pic}^{0}\left(X_{y}\right)$.

To prove (b), consider $\pi: X \longrightarrow W \subset S$. Assume that $V^{0}\left(X, Q+\operatorname{Pic}^{0}(S), \omega_{X}\right)$ is empty, then by Theorem 1.3.1.c, $H^{i}\left(X, \omega_{X} \otimes a^{*} Q \otimes \pi^{*} P\right)=0$ for all $i \geq 0$ and all $P \in \operatorname{Pic}^{0}(S)$. By Theorem 1.1.2, $H^{k}\left(W, R^{j} \pi_{*}\left(\omega_{X} \otimes a^{*} Q\right) \otimes P\right)=0$ for all $k$, and all $P \in \operatorname{Pic}^{0}(S)$. Therefore, by Lemma $2.1, R^{j} \pi_{*}\left(\omega_{X} \otimes a^{*} Q\right)=0$ for all $j$. In particular, for a general fiber $X_{w}$ of $\pi: X \longrightarrow W$,

$$
h^{0}\left(X_{w}, \omega_{X_{w}} \otimes a^{*} Q\right)=0
$$

Notice that $X_{w}$ is a finite union of general fibers of $X \rightarrow Y$ hence a finite union of varieties birational to $\tilde{K}$, therefore for $Q \in G$

$$
h^{0}\left(X_{w}, \omega_{X_{w}} \otimes a^{*} Q\right)=h^{0}\left(X_{w}, \omega_{X_{w}}\right)>0,
$$

which is a contradiction. We may therefore assume that $V^{0}\left(X, Q+\operatorname{Pic}^{0}(S), \omega_{X}\right)$ is nonempty.

The assertion (c) now follows since by Proposition 1.3.3, any isolated point $Q$ of $V^{0}\left(X, \operatorname{Pic}^{0}(A), \omega_{X}\right)$ must be such that $a^{*} Q=\mathcal{O}_{X}$.

Theorem 2.3. Let $a: X \longrightarrow A$ be a generically finite morphism from a smooth complex projective variety to an abelian variety. Then, the translates through the origin of the components of $V^{0}\left(X, \operatorname{Pic}^{0}(A), \omega_{X}\right)$ generate $\operatorname{Pic}^{0}(S)$.

Proof. Assume that translates through the origin of the components of $V^{0}:=$ $V^{0}\left(X, \operatorname{Pic}^{0}(A), \omega_{X}\right)$ do not generate $\operatorname{Pic}^{0}(S)$. Then there exists an abelian proper 
subvariety $T \subset \operatorname{Pic}^{0}(S)$ and a finite subgroup $G^{\prime}$ such that $V^{0} \subset T+G^{\prime}$. Consider the induced morphism

$$
\pi: X \longrightarrow T^{*}=: C,
$$

which factors through $X \stackrel{a}{\rightarrow} A \stackrel{p}{\rightarrow} S \rightarrow C$.

Let $Q \in \operatorname{Pic}^{\tau}(A)$ be any torsion element not contained in $T+G^{\prime}$. Then, $H^{0}\left(X, \omega_{X} \otimes a^{*} Q \otimes \pi^{*} P\right)=0$ for all $i \geq 0$ and all $P \in \operatorname{Pic}^{0}(C)$. By Theorem 1.3.1.c, $H^{i}\left(X, \omega_{X} \otimes Q \otimes \pi^{*} P\right)=0$ for all $0 \leq i \leq n$ and all $P \in \operatorname{Pic}^{0}(C)$.

Let $V \subset C$ be the image of $X$, and $\pi: X \longrightarrow V$ be the induced map. By Theorem 1.1.2 and the projection formula, it follows that

$$
H^{j}\left(V, R^{i} \pi_{*}\left(\omega_{X} \otimes Q\right) \otimes P\right)=0
$$

for all $i, j$ and $P \in \operatorname{Pic}^{0}(C)$. By Lemma 2.1, $R^{i} \pi_{*}\left(\omega_{X} \otimes Q\right)=0$ for all $i$.

If $\pi: X \longrightarrow V$ is generically finite, then $\pi_{*}\left(\omega_{X} \otimes Q\right)$ is clearly non-zero. We may therefore assume that $\pi$ has positive dimensional fibers.

Let $p$ be a point in $V$ and $X_{p}=\pi^{-1}(p)$ be the inverse image of $p$. For general $p \in V$ (not depending on $Q$ ), we have that

$$
H^{i}\left(X_{p}, \omega_{X_{p}} \otimes Q\right)=H^{i}\left(X_{p},\left(\omega_{X} \otimes Q\right) \otimes \mathcal{O}_{X_{p}}\right)=0 .
$$

Let $B$ be the connected component through the origin of the kernel of $A \longrightarrow C$. The image of $X_{p}$ in $A$ is contained in a translate of $B$ which we denote by $B_{p}$. Since $h^{0}\left(X_{p}, \omega_{X_{p}} \otimes Q\right)=0$ for all but finitely many $Q \in \operatorname{Pic}^{0}(B)$, it follows by Proposition 1.3.2 that $\kappa\left(X_{p}\right)=0$ and $X_{p} \longrightarrow B_{p}$ is birationally an étale map of abelian varieties.

On the other hand, by the weak addition formula

$$
\kappa\left(X_{p}\right)+\operatorname{dim}(V) \geq \kappa(X),
$$

so $\kappa\left(X_{p}\right)>0$ since $\kappa(X)=\operatorname{dim}(S)>\operatorname{dim}(V)$. This contradicts the assumption that $T$ is a proper subvariety of $\operatorname{Pic}^{0}(S)$. Therefore, $T=\operatorname{Pic}^{0}(S)$.

Corollary 2.4. In the above hypothesis, the dimension of the subgroup of $\operatorname{Pic}^{0}(X)$ generated by the translates through the origin of the components of $V^{0}\left(X, \operatorname{Pic}^{0}(A)\right.$, $\left.\omega_{X}\right)$ is equal to $\kappa(X)-\operatorname{dim}(X)+q(X)$.

Example [Ko3, 17.9.5]. Let $p: C \longrightarrow E$ be a degree 2 map from a genus 2 curve $C$ to an elliptic curve $E$. We may assume that $p_{*} \mathcal{O}_{C}=\mathcal{O}_{E} \oplus L^{-1}$, for an appropriate $L \in \operatorname{Pic}(E)$ such that $L^{\otimes 2}=\mathcal{O}_{E}(B)$ where $B$ is the branch locus. Let $\tilde{F} \longrightarrow F$ be a degree 2 étale map of elliptic curves such that $e_{*} \mathcal{O}_{\tilde{F}}=\mathcal{O}_{F} \oplus P$ with $P^{\otimes 2} \cong \mathcal{O}_{F}$. Define

$$
X:=\tilde{F} \times C /<\left(i_{\tilde{F}} \times i_{C}\right)>.
$$

Here $i_{\tilde{F}}$ and $i_{C}$ denote the involutions on $\tilde{F}$ and $C$ respectively. We have that for $a: X \longrightarrow F \times E$,

$$
a_{*}\left(\omega_{X}\right) \cong\left(\mathcal{O}_{F} \otimes \mathcal{O}_{E}\right) \oplus(P \otimes L) .
$$

(Pull backs have been omitted.) It follows that Iitaka fibration has image $E$ and

$$
V^{0}\left(X, \operatorname{Pic}^{0}(F \times E), \omega_{X}\right)=\left\{\mathcal{O}_{F \times E}\right\} \cup\left(P+\operatorname{Pic}^{0}(E)\right) .
$$

In particular $V^{0}\left(X, \operatorname{Pic}^{0}(E), \omega_{X}\right)$ does not generate $\operatorname{Pic}^{0}(E)$. 
Corollary 2.5. Let $X$ be any variety of maximal Albanese dimension. $\kappa(X)$ is invariant under smooth deformations.

Proof. Let $\Delta$ be an open neighborhood of a point 0 of a smooth projective curve, $\delta: \mathcal{X} \longrightarrow \Delta$ be a smooth projective morphism with connected smooth fibers, $\mathcal{X}_{0}:=\delta^{-1}(0)$ be a closed fiber of $\delta$ such that $\mathcal{X}_{0}=X$. Since $q(X)$ is deformation invariant, for all $t \in \Delta, A_{t}:=\operatorname{Alb}\left(\mathcal{X}_{t}\right)$ is an abelian variety of dimension $q(X)$.

Let $P \in \operatorname{Pic}^{0}(X)$ such that $P^{\otimes m}=\mathcal{O}_{X}, \mathcal{P}$ be a section of $\operatorname{Pic}^{0}(\mathcal{X} / \Delta)$ such that $P=P_{0}:=\left.\mathcal{P}\right|_{\mathcal{X}_{0}}, P_{t}:=\left.\mathcal{P}\right|_{\mathcal{X}_{t}} \in \operatorname{Pic}^{0}\left(A_{t}\right)$ satisfies $P_{t}^{\otimes m}=\mathcal{O}_{\mathcal{X}_{t}}$. Let

$$
\tilde{\mathcal{X}}_{t}:=\operatorname{Spec}\left(\oplus_{i=0}^{m-1} P_{t}^{\otimes i}\right)
$$

be the corresponding étale cyclic cover of degree $m$. We have that the quantity

$$
h^{0}\left(\tilde{\mathcal{X}}_{t}, \omega_{\tilde{\mathcal{X}}_{t}}\right)=\sum_{i=0}^{m-1} h^{0}\left(\omega_{\mathcal{X}_{t}} \otimes P_{t}^{\otimes i}\right)
$$

is constant. However the functions $h^{0}\left(\mathcal{X}_{t}, \omega_{\mathcal{X}_{t}} \otimes P_{t}^{\otimes i}\right)$ are upper semicontinuous in $t \in \Delta$, and hence also constant.

Let $\bar{T}^{i}$ denote the translates of the components $T^{i}$ of $V^{0}\left(X, \operatorname{Pic}^{0}(A), \omega_{X}\right)$ through the origin. The subvarieties $T^{i}$ are determined by their torsion points (cf. Theorem 1.3.1.d). In particular, recall that $\operatorname{Pic}^{0}\left(\mathcal{X}_{t}\right) \cong H^{1}\left(\mathcal{X}_{t}, \mathcal{O}_{\mathcal{X}_{t}}^{*}\right) / H_{1}\left(\mathcal{X}_{t}, \mathbb{Z}\right)$ and the subvarieties $\bar{T}_{t}^{i}$ are determined by the corresponding vector subspaces of $H_{1}\left(\mathcal{X}_{t}, \mathbb{Q}\right)$. We remark that given two $\mathbb{Q}$ vector subspaces $W_{t}^{i} \subset H_{1}\left(\mathcal{X}_{t}, \mathbb{Q}\right)$ continuous in the parameter $t$, then $\operatorname{dim}\left(W_{t}^{1} \cap W_{t}^{2}\right)$ and $\operatorname{dim}\left(W_{t}^{1}+W_{t}^{2}\right)$ are constant.

From the above discussion, it follows that there exist subvarieties $T_{t}^{i}$ of $\operatorname{Pic}^{0}\left(A_{t}\right)$, which are smooth deformations of $T_{0}^{i}$ such that $V^{0}\left(\mathcal{X}_{t}, \operatorname{Pic}^{0}\left(A_{t}\right), \omega \mathcal{X}_{t}\right)=\cup T_{t}^{i}$. Moreover, for any set of indices $I$, the quantities

$$
\operatorname{dim}\left(\bigcap_{i \in I} \bar{T}_{t}^{i}\right) \quad \text { and } \operatorname{dim}\left(\sum_{i \in I} \bar{T}_{t}^{i}\right)
$$

are constant. In particular the quantity

$$
\operatorname{dim}\left(V^{0}\left(\mathcal{X}_{t}, \operatorname{Pic}^{0}\left(A_{t}\right), \omega_{\mathcal{X}_{t}}\right)\right)=\kappa\left(\mathcal{X}_{t}\right)+q\left(\mathcal{X}_{t}\right)-\operatorname{dim}\left(\mathcal{X}_{t}\right)
$$

is constant and hence $\kappa\left(\mathcal{X}_{t}\right)$ is also constant.

\section{Pluricanonical maps of varieties of maximal Albanese dimension}

We will keep the notation of the preceding sections. In particular $X$ will be a smooth projective variety with maximal Albanese dimension and $a: X \longrightarrow A$ will denote a generically finite morphism to an abelian variety.

Lemma 3.1. Let $E$ be an a-exceptional effective divisor on $X$. If $\mathcal{O}_{X}(E) \otimes P$ is effective, then $P=\mathcal{O}_{X}$.

Proof. If $\operatorname{dim}(X)=1$, then $E=0$ and the assertion is clear. If $\operatorname{dim}(X)>1$, pick $H_{A}$ a sufficiently ample divisor on $A$ and let $H=a^{*} H_{A}$ be the corresponding nef and big divisor on $X$. Choosing $H_{A}$ appropriately, we may assume that $H$ is a 
smooth divisor in $X$. We may also assume that $h^{0}\left(X, \mathcal{O}_{X}(E-H) \otimes P\right)=0$ for all $P \in \operatorname{Pic}^{0}(X)$. If $\operatorname{dim}(X) \geq 2$, from the exact sequence of sheaves

$$
0 \longrightarrow \mathcal{O}_{X}(E-H) \otimes P \longrightarrow \mathcal{O}_{X}(E) \otimes P \longrightarrow \mathcal{O}_{H}(E) \otimes P \longrightarrow 0,
$$

we have an injection $H^{0}\left(X, \mathcal{O}_{X}(E) \otimes P\right) \hookrightarrow H^{0}\left(H, \mathcal{O}_{H}(E) \otimes P\right)$ for all $P \in \operatorname{Pic}^{0}(X)$. Similarly, there is an exact sequence of sheaves

$$
0 \longrightarrow \mathcal{O}_{X}(-H) \otimes P \longrightarrow \mathcal{O}_{X} \otimes P \longrightarrow \mathcal{O}_{H} \otimes P \longrightarrow 0 .
$$

Since $H$ is nef and big,

$$
h^{i}\left(X, \mathcal{O}_{X}(-H) \otimes P\right)=h^{n-i}\left(X, \omega_{X}(H) \otimes P^{*}\right)=0 \quad \text { for all } i<n .
$$

Therefore, $h^{0}\left(X, \mathcal{O}_{X} \otimes P\right)=h^{0}\left(H, \mathcal{O}_{H} \otimes P\right)=0$ for $P \neq \mathcal{O}_{X}$ and $h^{0}\left(X, \mathcal{O}_{X}\right)=$ $h^{0}\left(H, \mathcal{O}_{H}\right)=1$. Clearly, for a general choice of $H$, we have that $H$ is of maximal Albanese dimension, and $\left.E\right|_{H}$ is an $\left.a\right|_{H}$-exceptional divisor. Repeating the above procedure, by successively intersecting appropriate divisors pulled back from $A$, one obtains a curve $C \subset X$ such that

i) If $h^{0}\left(X, \mathcal{O}_{X}(E) \otimes P\right)>0$ then $h^{0}\left(C, \mathcal{O}_{C}(E) \otimes P\right)=h^{0}\left(C, \mathcal{O}_{C} \otimes P\right)>0$,

ii) If $h^{0}\left(C, \mathcal{O}_{C} \otimes P\right)>0$ then $P=\mathcal{O}_{X}$.

It follows that if $h^{0}\left(X, \mathcal{O}_{X}(E) \otimes P\right)>0$, then $P=\mathcal{O}_{X}$.

Lemma 3.2. Let $D$ be an irreducible reduced divisor on $X$ which is not $a: X \longrightarrow A$ exceptional, $H$ a Cartier divisor on $X$ which is numerically trivial on the general fiber of $X \longrightarrow S$. Then, $D$ is not contained in the base locus of $\left|m K_{X}+H\right|$ for infinitely many values of $m$.

Proof. [Ko3, 17.6.1].

Let $\left|m K_{X}\right|=F_{m}+\left|M_{m}\right|$, where $B s\left|M_{m}\right|$ contains no divisors. We remark that, since $X$ is of maximal Albanese dimension, we may assume that $K_{X}$ is effective.

Theorem 3.3. If $X$ is of general type, i.e. $\kappa(X)=\operatorname{dim}(X)$. Then for any integer $s \geq 3, F_{s}$ is a-exceptional. $\left|5 K_{X}\right|$ defines a generically finite rational map and $\left|(5 \operatorname{dim}(X)+1) K_{X}\right|$ defines a birational map.

Proof. For any fixed divisor $D$ on $X$, then there exists an $m_{0}$ such that $\left|m K_{X}-D\right| \neq$ $\emptyset$ for all $m \geq m_{0}$. Let $R$ be an irreducible divisor of $X$ which is not $a$-exceptional. By Lemma 3.2, $R \not \subset B s\left|m K_{X}-D\right|$ for infinitely many $m \gg 0$.

Step 1. $F_{s}$ is exceptional for all $s \geq 3$.

It is easy to see that $F_{s}$ being $a$-exceptional is a condition which is independent of the particular birational model of $X$ under consideration. $F_{1} \subset R_{a}$, the ramification divisor of $a: X \longrightarrow A$, contains at most finitely many non $a$ exceptional components, which we denote by $R_{i}$. Fix positive integers $m_{i}$ such that $R_{i} \not \subset B s\left|m_{i} K_{X}\right|$, then $R_{i} \not \subset B s\left|\lambda m_{i} K_{X}\right|$ for any integer $\lambda>0$. It follows that for $m_{0}=\prod m_{i}, F_{m_{0}}$ is $a$-exceptional.

Next, fix an ample divisor $H$ on $A$, then $a^{*} H$ is nef and big on $X$. Let $K_{X}$ be a canonical divisor. Let $r$ be the multiplicity of $K_{X}$ at $R$. We distinguish two cases. Case 1. $r=1$.

By Lemma 3.2, there exists a positive integer $t$ such that the base locus of $\left|t K_{X}-a^{*} H\right|$ doesn't contain $R$. Let $B$ be an general element of $\left|(s-2)\left(t K_{X}-a^{*} H\right)\right|$. 
By replacing $X$ with an appropriate birational model, we may assume that $R$ is smooth and $B+R$ has normal crossing support and $B$ does not contain $R$. Define

$$
M:=\mathcal{O}_{X}\left((s-2) K_{X}-\left\lfloor\frac{B}{t}\right\rfloor\right) \equiv\left(\frac{s-2}{t}\right) a^{*} H+\left\{\frac{B}{t}\right\} .
$$

Consider the exact sequence

$$
0 \rightarrow \omega_{X} \otimes M \rightarrow \omega_{X} \otimes M(R) \rightarrow \omega_{R} \otimes M \rightarrow 0 .
$$

Since $a^{*} H$ is nef and big and $\left\{\frac{B}{t}\right\}$ is klt, one sees that $H^{1}\left(X, \omega_{X} \otimes M\right)=0$. The divisor $R$ is not $a$-exceptional, so $K_{R}$ is effective and $\left.\left(a^{*} H\right)\right|_{R}$ is nef and big. $R$ is not contained in the support of $B$, so the $\mathbb{Q}$ divisor $\left\{\frac{1}{t}\left(\left.B\right|_{R}\right)\right\}$ is also klt. By Theorem 1.1.1.e, $H^{0}\left(R, \omega_{R} \otimes M\right) \neq 0$. Therefore, there is a divisor in $\left|K_{X}+M+R\right|$ not containing $R$. This gives a divisor in $\left|s K_{X}\right|=\left|K_{X}+M+R+\left(K_{X}-R\right)+\left\lfloor\frac{B}{t}\right\rfloor\right|$ not containing $R$.

Case 2. $r \geq 2$.

There exists positive integers $t, m_{0}$ such that $R \not \subset B s\left|t K_{X}-a^{*} H\right|$, and $R \not \subset$ $B s\left|m_{0} K_{X}\right|$. For any integer $s \geq 2$ let $K^{\prime}:=(s-1) K_{X}-R$. Consider the following linear series

$$
\left|m K^{\prime}-m_{0} a^{*} H\right|=\left|\left((s-1) m-\frac{m}{r}-m_{0} t\right) K_{X}+\frac{m}{r}\left(K_{X}-r R\right)+m_{0}\left(t K_{X}-a^{*} H\right)\right| .
$$

It follows that for $m$ divisible by $m_{0} r, R$ is not in the base locus of $\left|m K^{\prime}-m_{0} a^{*} H\right|$. Choose a general $B \in\left|m K^{\prime}-m_{0} a^{*} H\right|$ and define

$$
M:=\mathcal{O}_{X}\left(K^{\prime}-\left\lfloor\frac{B}{m}\right\rfloor\right) \equiv \frac{m_{0}}{m} a^{*} H+\left\{\frac{B}{m}\right\} .
$$

An argument similar to the one in the previous case again shows that $R$ is not in the base locus of $\left|s K_{X}\right|$.

Step 2. $\left|5 K_{X}\right|$ defines a generically finite map.

Replacing $X$ by an appropriate birational model, we may assume that $\left|M_{3}\right|$ is base point free. Let $D$ be a general member of $M_{3}$ and $\Delta$ be the image of $D$ in $A$. If $\Delta$ is not of general type, then there exists an ample line bundle $H$, a semipositive line bundle $L$ and a positive integer $s$ such that $\Delta \cdot L^{s} \cdot H^{n-s-1}=0$ and $L^{s} \cdot H^{n-s}>0$. It follows that also $D \cdot\left(a^{*} H\right)^{n-s-1} \cdot\left(a^{*} L\right)^{s}=0$.

Since $X$ is of general type, there exists a rational number $\epsilon>0$ such that $K_{X}-\epsilon\left(a^{*} H\right)$ is an effective $\mathbb{Q}$-divisor. Therefore,

$$
K_{X} \cdot\left(a^{*} H\right)^{n-s-1} \cdot\left(a^{*} L\right)^{s} \geq \epsilon\left(a^{*} H\right)^{n-s} \cdot\left(a^{*} L\right)^{s}>0 .
$$

Since $K_{X}-D \equiv F_{3}$ is $a$-exceptional, it follows that

$$
D \cdot\left(a^{*} H\right)^{n-s-1} \cdot\left(a^{*} L\right)^{s}=K_{X} \cdot\left(a^{*} H\right)^{n-s-1} \cdot\left(a^{*} L\right)^{s}>0 .
$$

This is a contradiction. We may therefore assume that $\Delta$ and hence $D$ are of general type. It is well known that for an appropriate desingularization $\Delta^{\prime}$ of $\Delta$, the linear series $\left|K_{\Delta^{\prime}}\right|$ defines a generically finite rational map. Therefore, we may assume that $\left|K_{D}\right|$ defines a generically finite rational map. Let $B \in\left|m K_{X}-a^{*} H\right|$, and $M:=\mathcal{O}_{X}\left(K_{X}-\left\lfloor\frac{B}{m}\right\rfloor\right) \equiv \frac{a^{*} H}{m}+\left\{\frac{B}{m}\right\}$. We may assume that $B$ has normal crossings support and does not contain $D$. Consider the exact sequence of sheaves

$$
0 \longrightarrow \omega_{X} \otimes M \longrightarrow \omega_{X} \otimes M(D) \longrightarrow \omega_{D} \otimes M \longrightarrow 0,
$$

which is also exact on global sections. 
Claim. There exist $m, B$ such that $M$ is effective.

Fix $m_{1}$ such that $\left|m_{1} K_{X}-a^{*} p^{*} H_{S}\right|$ is non empty. Let $B_{1} \in\left|m_{1} K_{X}-a^{*} p^{*} H_{S}\right|$. Let $m:=m_{1}+m_{2}$ and $B:=B_{1}+m_{2} K_{X} \in\left|m K_{X}-a^{*} p^{*} H_{S}\right|$. Let $\Gamma$ be any prime divisor, and $b_{1}, k$ be the multiplicities of $B_{1}, K_{X}$ along $\Gamma$. For $m_{2} \gg 0$, we have that

$$
\left\lfloor\frac{b_{1}+k m_{2}}{m}\right\rfloor=\left\lfloor\frac{b_{1}}{m_{1}+m_{2}}+k \frac{m_{2}}{m_{1}+m_{2}}\right\rfloor \leq k .
$$

Since there are only finitely many components of $B+K_{X}$, for $m_{2} \gg 0$, we have $\left\lfloor\frac{B}{m}\right\rfloor \prec K_{X}$, and the claim follows.

It is easy to see that $\left.M\right|_{D}$ is also effective. Therefore, $\left|K_{X}+D+M\right|$ restricted to $D$ defines a generically finite rational map. If $\left|K_{X}+D+M\right|$ does not define a generically finite rational map, then the closure of the image of $X$ which we denote by $Y \subset \mathbb{P}=\mathbb{P}\left(H^{0}\left(X, \omega_{X} \otimes M(D)\right)\right)$, must be dominated by $D$. This is however impossible since by Theorem 1.1.1.e $h^{0}\left(X, \omega_{X} \otimes M\right)>0$, and hence $\left|K_{X}+D+M\right|$ contains non-trivial sections vanishing on $D$, i.e. there is a hyperplane section of $\mathbb{P}$ containing the image of $D$ but not containing $Y$. Finally, the assertion follows from the inclusion of sheaves

$$
\omega_{X} \otimes M(D) \hookrightarrow \omega_{X}^{\otimes 5} .
$$

By [Ko1, Theorem 8.1], $\left|(5 \operatorname{dim}(X)+1) K_{X}\right|$ defines a birational map.

Theorem 3.4. $\left|6 K_{X}\right|$ defines rational map with image of dimension $\kappa(X)$, and $\left|(6 \kappa(X)+2) K_{X}\right|$ defines the stable canonical map.

Proof. Let $H_{A}, H_{S}$ be the pull backs of sufficiently ample divisors on $A, S$ respectively. For any $P \in \operatorname{Pic}^{0}(X)$, let

$$
\left|m K_{X}+P\right|=\left|M_{m, P}\right|+F_{m, P},
$$

where $B s\left|M_{m, P}\right|$ contains no divisor.

Step 1. Let $S \longrightarrow S^{\prime}$ be any surjective map of abelian varieties such that $y:=$ $\operatorname{dim}(S)>y^{\prime}:=\operatorname{dim}\left(S^{\prime}\right)$ and $s^{\prime}: X \rightarrow S^{\prime}$ be the induced map. Then, for any divisor $D \in\left|M_{4}\right|, D$ is not $s^{\prime}$-vertical.

By Lemma 1.3.4, the linear series $\left|2 K_{X}+P\right|$ is nonempty for all $P \in \operatorname{Pic}^{0}(S)$. Let $Z$ be an irreducible component of $H_{S^{\prime}}^{y^{\prime}}$, ie a general fiber of $s^{\prime}: X \rightarrow S^{\prime}$. For all $P \in \operatorname{Pic}^{0}(S)$ one has a map of linear series

$$
\left|2 K_{X}+P\right| \times\left|2 K_{X}-P\right| \longrightarrow\left|4 K_{X}\right| .
$$

It follows that

$$
F_{4} \subset \bigcap_{P \in \operatorname{Pic}^{0}(S)}\left(F_{2, P}+F_{2,-P}\right) .
$$

Claim 1. $\left\lceil\frac{F_{4}}{2}\right\rceil \prec F_{2, P}$ for general $P \in \operatorname{Pic}^{0}(S)$.

Let $V \subset \operatorname{Pic}^{0}(S)$ be an open set such that $h^{0}\left(X, \omega_{X}^{\otimes 2} \otimes P\right)$ is constant. Let $R$ be any component of $F_{4}$ of multiplicity $r$, then $\left\lceil\frac{r}{2}\right\rceil R \prec F_{2, P}$ for all $P \in U \subset \operatorname{Pic}^{0}(S)$ where $U$ is a Zariski dense subset such that $U \cup-U=\operatorname{Pic}^{0}(S)$. Since the condition $\left\lceil\frac{r}{2}\right\rceil R \prec B s\left|2 K_{X}+P\right|$ is Zariski closed in $V$, it follows that $\mathcal{O}_{X}\left(2 K_{X}-\left\lceil\frac{r}{2}\right\rceil R\right) \otimes P$ is effective for all $P \in V \subset \operatorname{Pic}^{0}(S)$. Since this holds for all components of $F_{4}$, the claim follows. 
Claim 2. $\operatorname{ker}\left(\operatorname{Pic}^{0}(S) \rightarrow \operatorname{Pic}^{0}(Z)\right)$ is a proper closed subvariety of $\operatorname{Pic}^{0}(S)$.

Let $V$ be the image of $Z$ in $S . V$ is a translate of $\operatorname{ker}\left(S \rightarrow S^{\prime}\right)$. There exists infinitely many $P \in \operatorname{Pic}^{0}(S)$ such that $\left.P\right|_{V} \neq \mathcal{O}_{V}$. Hence pulling back to $Z$, we have $\left.P\right|_{Z} \neq \mathcal{O}_{Z}$.

Claim 3. $\left.\left(2 K_{X}-\left\lceil\frac{F_{4}}{2}\right\rceil\right)\right|_{Z}$ is not a $\left.\right|_{Z}$-exceptional.

By Claim 1, $\left.\left(2 K_{X}-\left\lceil\frac{F_{4}}{2}\right\rceil+P\right)\right|_{Z}$ is effective and for general $P \in \operatorname{Pic}^{0}(S)$. By Claim 2, we may assume that $\left.P\right|_{Z} \neq \mathcal{O}_{Z}$ for general $P \in \operatorname{Pic}^{0}(S)$. Since $\left.a\right|_{Z}: Z \longrightarrow a(Z)$ is generically finite, the claim follows by Lemma 3.1.

Claim 3 implies that $2 K_{X}-\left\lceil\frac{F_{4}}{2}\right\rceil$ is not $s^{\prime}$-vertical (otherwise $\left.\left(2 K_{X}-\left\lceil\frac{F_{4}}{2}\right\rceil\right)\right|_{Z}$ $\left.=\mathcal{O}_{Z}\right)$. From the inclusion of linear series

$$
\left|2 K_{X}-\left\lceil\frac{F_{4}}{2}\right\rceil\right| \times\left|2 K_{X}-\left\lceil\frac{F_{4}}{2}\right\rceil\right| \stackrel{+2\left\lceil\frac{F_{4}}{2}\right\rceil-F_{4}}{\longrightarrow}\left|4 K_{X}-F_{4}\right|
$$

it follows that $4 K_{X}-F_{4}=M_{4}$ is also not $s^{\prime}$-vertical.

Step 2. Let $D$ be a general member of $M_{4}$, and $\Delta$ be its image in $S$. Then $\Delta$ is of general type.

If $\Delta$ is not of general type, then $\Delta$ is vertical for an appropriate projection $S \longrightarrow S^{\prime}$. By Step 1 this is impossible.

Step 3. $\left|6 K_{X}\right|$ defines a rational map whose image is of dimension $\kappa(X)$.

Let $\tilde{\Delta}$ be an appropriate desingularization of $\Delta$. The linear series $\left|K_{\tilde{\Delta}}\right|$ defines a generically finite rational map. Replacing $X$ by an appropriate birational model, we may assume that $\left|M_{4}\right|$ is free and hence $D$ is a smooth subvariety that maps onto $\tilde{\Delta}$.

Fix an ample divisor $H_{S}$ on $S$. Let $B \in\left|m K_{X}-a^{*} p^{*} H_{S}\right|$ be a general member. Replacing $X$ by an appropriate birational model, we may assume that $B$ has normal crossings support. Define

$$
M:=\mathcal{O}_{X}\left(K_{X}-\left\lfloor\frac{B}{m}\right\rfloor\right) \equiv \frac{a^{*} p^{*} H_{S}}{m}+\left\{\frac{B}{m}\right\} .
$$

As in the proof of the previous theorem, we may assume that $M,\left.M\right|_{D}$ are effective, and $B$ does not contain $D$. Consider the exact sequence:

$$
0 \longrightarrow \omega_{X} \otimes M \longrightarrow \omega_{X} \otimes M(D) \longrightarrow \omega_{D} \otimes M \longrightarrow 0 .
$$

By [Ko3,Theorem 10.19], this is exact on global sections. Sections of $\omega_{D} \otimes M$ lift to sections of $\omega_{X} \otimes M(D)$. Since $\mathcal{O}_{D}\left(K_{D / \tilde{\Delta}}\right) \otimes M$ is effective. It follows that $\omega_{D} \otimes M$ also defines a rational map with image of dimension at least $\kappa(X)-1=\operatorname{dim}(W)-1$. By Theorem 1.1.1.e, $\left|K_{X}+M\right|$ is non empty. An argument similar to the one in the proof of Theorem 3.3 shows that $\left|K_{X}+M+D\right|$ defines a rational map with image of dimension at least $\kappa(X)$. The assertion follows from the inclusion of sheaves

$$
\omega_{X} \otimes M(D) \hookrightarrow \omega_{X}^{\otimes 6}
$$


Step 4. $\left|(6 \kappa(X)+2) K_{X}\right|$ is the stable canonical map.

We may assume that $\left|6 K_{X}\right|=\left|M_{6}\right|+F_{6}$ and $M_{6}$ is free. Let $M$ be defined as in step 3. $\varphi_{6}$ factors as $X \longrightarrow Y \longrightarrow Y^{\prime}:=\varphi_{6}(X)$. Pick $D_{1}, \ldots, D_{\kappa}$ general sections of $M_{6}$, with $\kappa=\kappa(X)$. Let $X_{i}:=D_{1} \cap \ldots \cap D_{i}$. Then $X_{\kappa}=D_{1} \cap \ldots \cap D_{\kappa}$ is the union of $\operatorname{deg}\left(Y \longrightarrow Y^{\prime}\right)$ fibers of $X \longrightarrow Y$ which we denote by $F_{t}$. We must show that sections of $\left|(6 \kappa(X)+2) K_{X}\right|$ separate these fibers. Let $g_{i}: X_{i} \rightarrow \bar{X}_{i}$ be the maps induced by $X \rightarrow Y^{\prime}$. By [Ko3, Theorem 10.19], we have that

$$
H^{1}\left(X_{i}, \omega_{X_{i}} \otimes M \otimes M_{6}^{\otimes \kappa-i-1}\right) \longrightarrow H^{1}\left(X_{i}, \omega_{X_{i}} \otimes M \otimes M_{6}^{\otimes \kappa-i}\right)
$$

is injective for all $i \leq \kappa-1$. Therefore, the exact sequences

$$
0 \rightarrow \omega_{X_{i}} \otimes M \otimes M_{6}^{\otimes \kappa-i-1} \rightarrow \omega_{X_{i}} \otimes M \otimes M_{6}^{\otimes \kappa-i} \rightarrow \omega_{X_{i+1}} \otimes M \otimes M_{6}^{\otimes \kappa-i-1} \rightarrow 0,
$$

are exact on global sections. It follows that

$$
H^{0}\left(X, \omega_{X} \otimes M \otimes M_{6}^{\otimes \kappa(X)}\right) \longrightarrow H^{0}\left(X_{\kappa}, \omega_{X_{\kappa}} \otimes M\right)
$$

is surjective. The assertion now follows since $\omega_{X} \otimes M \otimes M_{6}^{\otimes \kappa(X)}$ is a subsheaf of $\omega_{X} \otimes(6 \kappa(X)+2), H^{0}\left(X_{\kappa}, \omega_{X_{\kappa}} \otimes M\right)=\oplus H^{0}\left(F_{t}, \omega_{F_{t}} \otimes M\right)$, and by Theorem 1.1.1.e, $H^{0}\left(F_{t}, \omega_{F_{t}} \otimes M\right) \neq 0$.

\section{REFERENCES}

[AS] U. Angehrn, Y.-T. Siu, Effective freeness and point separation for adjoint bundles, Inv. Math. 122 (1995), no. 2, 291-308.

[CH] J. A. Chen, C. D. Hacon, Characterization of Abelian Varieties, Preprint alg-geom9903184 (1999).

[GL1] M. Green, R. Lazarsfeld, Deformation theory, generic vanishing theorems, and some conjectures of Enriques, Catanese and Beauville, Invent. Math. 90 (1987), 389-407.

[GL2] Higher obstruction to deforming cohomology groups of line bundles, Jour. Amer. Math. Soc. 4 (1991), 87-103.

[EL1] L. Ein, R. Lazarsfeld, Singularities of theta divisors, and birational geometry of irregular varieties, Jour. AMS 10 (1997), no. 1, 243-258.

[EL2] Unpublished personal communication.

[EV1] H. Esnault, E. Viehweg, Revêtements cyclique II, Géométrie Algèbrique et Applications II, La Rábida. Herman, Paris (1987), 81-94.

[Ka1] Y. Kawamata, Characterization of Abelian Varieties, Comp. Math. 43 (1981), 253-276.

[Ka2] Minimal models and the Kodaira dimension of algebraic fiber spaces, Jour. Reine Angew. Math. 363 (1985), 1-46.

[Ko1] J. Kollár, Higher direct images of dualizing sheaves I, Ann. Math. 123 (1986), 11-42.

[Ko2] - Higher direct images of dualizing sheaves II, Ann. Math. 124 (1987).

[Ko3] Shafarevich Maps and Automorphic Forms, Princeton University Press, 1995.

$[\mathrm{M}] \quad$ S. Mukai, Duality between $D(X)$ and $D(\hat{X})$, with application to Picard sheaves, Nagoya math. J. 81 (1981), 153-175.

[S] C. Simpson, Subspaces of moduli spaces of rank one local systems, Ann. Sci. École Norm. Sup. (4) 26 (1993), no. 3, 361-401.

Jungkai Alfred Chen, Department of Mathematics, National Chung Cheng University, Ming Hsiung, Chia Yi, 621, Taiwan

E-mail address: jkchen@math.ccu.edu.tw

Christopher Derek Hacon, Department of Mathematics, University of Utah, 155 South 1400 East JWB 233, Salt Lake City, UT 84112, USA

E-mail address: chhacon@math.utah.edu 